\title{
Electrical storm reversible by isoproterenol infusion in a striking case of early repolarization
}

\author{
Anne Bernard • Olivier Genée • Caroline Grimard • \\ Frédéric Sacher • Laurent Fauchier • Dominique Babuty
}

Received: 17 October 2008 / Accepted: 14 November 2008/Published online: 19 January 2009

(C) The Author(s) 2009. This article is published with open access at Springerlink.com

\begin{abstract}
A 40-year-old woman was referred to intensive care unit after recurrent ventricular fibrillation. She was free of cardiac medical history or medications. The resting ECG displayed an extended early repolarization in the inferior leads and all the precordial leads. Incessant ventricular fibrillations justified a treatment by intravenous amiodarone associated with general anaesthesia and mechanical ventilation without success on ventricular fibrillation. Because of a low heart rate intravenous isoproterenol infusion was initiated. Isoproterenol infusion was associated with heart rate acceleration and a decrease in $\mathrm{J}$ point elevation and the disappearance of ventricular fibrillation episodes. No cardiac disease was documented and the patient was implanted of a single chamber ICD. Six months later the patient was free of syncope and ventricular fibrillation on ICD memory. This case report demonstrates the usefulness and efficiency of the isoproterenol infusion to eliminate recurrent ventricular fibrillation in patients with early repolarization.
\end{abstract}

Keywords Sudden death · Ventricular fibrillation . Early repolarization $\cdot$ Electric storm $\cdot$ Isoproterenol

Early repolarization, usually considered a benign abnormality $[1,2]$, has recently been shown to be involved in

\footnotetext{
A. Bernard · O. Genée · C. Grimard · L. Fauchier · D. Babuty $(\bowtie)$ Cardiology Department Hospital Trousseau Tours University, Tours, France

e-mail: d.babuty@chu-tours.fr

F. Sacher

Cardiology Department Hospital du Haut Lévéque PESSAC

Bordeaux University,

PESSAC, France
}

primary ventricular fibrillation $[3,4,5]$. We reported a case of ventricular electrical storm in a patient with a major early repolarization which was successfully treated by isoproterenol infusion.

A 40-year-old woman was referred to the Emergency Room of a community hospital for recent recurrent syncopes. She had been examined three days earlier by her general practitioner who had found no cardiac or neurological abnormalities, and the resting ECG displayed early repolarization pattern extended in all the precordial leads (particularly in V2) as well as inferior leads, together with positive $\mathrm{T}$ waves (Fig. 1(a)). She had a history of epilepsy during her childhood, which was treated with antiepileptic drugs until the age of ten. There was no family history of sudden death. She reported that she had been suffering from acute gastroenteritis for a week, but she had received no medication and was free of chest pain. In the emergency room, new syncopes occurred, and ECG monitoring revealed numerous ventricular ectopic beats followed by ventricular fibrillation (VF) (Fig. 1(b)). Several VF episodes occurred which always started with the same sequence: a premature beat with major early repolarization was followed by a premature ventricular beat (left bundle branch block pattern and left axis with short coupling interval $320 \mathrm{~ms}$ ) initiating VF (Fig. 2(a,b). Sinus rhythm was restored by electric cardioversion (200 J biphasic). The evolution was marked by repetitive VF, justifying general anaesthesia associated with mechanical ventilation support without effect on VF. Twenty five external electric cardioversions were delivered over two hours' time despite the infusion of amiodarone (150 mg I.V), magnesium ( $2 \mathrm{~g}$ I.V) and potassium. She was not given an epinephrine infusion. Once haemodynamics had been stabilized and normal sinus rhythm restored, the patient was transferred to the cardiac ICU. 
Fig. 1 (a) ECG recorded $48 \mathrm{~h}$ before electrical storm showed early repolarization in V2-V6 leads. (b) Occurrence of premature ventricular contraction and ventricular fibrillation. (c) Resting ECG between two ventricular fibrillations displayed major $\mathrm{J}$ point and ST elevation in V2V6 leads
(A)
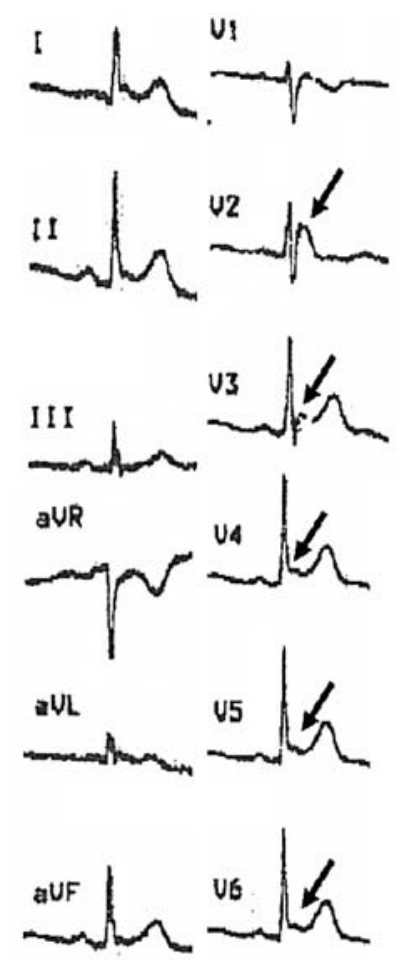

(C)

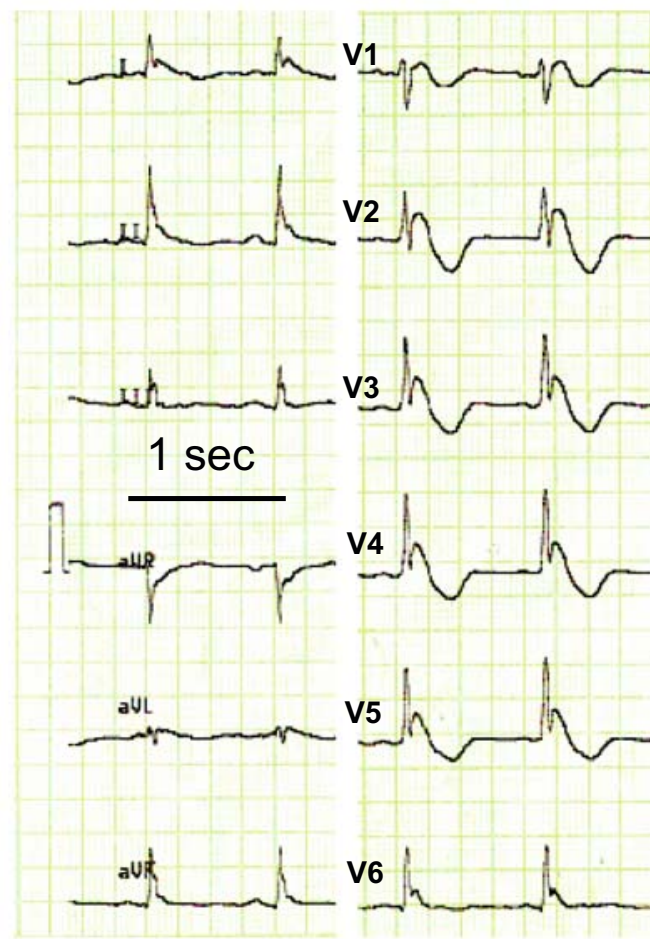

(B)

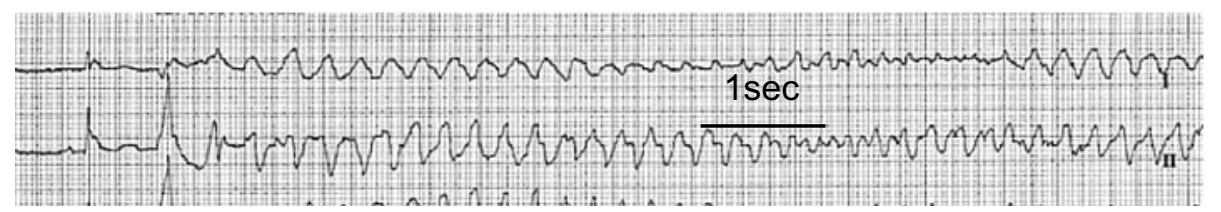

On admission blood pressure was $90 / 50 \mathrm{mmHg}$, heart rate $56 \mathrm{bpm}$, temperature $37^{\circ} \mathrm{C}$. Physical examination was unremarkable, with no cardiac murmur or abnormal heart sound. Intercritical electrocardiogram showed now sinus rhythm with early repolarization characterized by $4 \mathrm{~mm}$ prominent $\mathrm{J}$ wave and following down sloping ST elevation with negative $\mathrm{T}$ waves, in most of the precordial leads as well in the inferior leads to a lesser extend (Fig. 1(c)). QT interval was normal $(380 \mathrm{~ms})$. Transthoracic echocardiography revealed normal left ventricle size, and the left ventricular ejection fraction was $45 \%$. Initial laboratory findings revealed hypokalemia $(2.9 \mathrm{mmol} / \mathrm{l})$, normal magnesium level, no hypercalcemia, and normal troponin and CPK enzymes. Blood toxicological screening was negative for common drugs. Because of relative slow heart rhythm (about 50-60 beats / $\mathrm{min}$ ) and the ineffectiveness of amiodarone in preventing recurrence of VF (18 additional electric cardioversions), immediate treatment included stopping the amiodarone infusion and initiating intravenous isoproterenol infusion to accelerate the heart rate above $110 \mathrm{bpm}$. (from one to three micrograms/min). Twelve-lead ECG was continuously recorded during the isoproterenol infusion. Half an hour after starting isoproterenol infusion, sinus rhythm was about $110-120$ beats / min, the VF episodes disappeared, and the repolarization tended to be normalized (Fig. 3). The recurrence of VF was associated with the elevation of the J-point. Because ventricular arrhythmias and early repolarization reappeared on ECG whenever the isoproterenol infusion was slowed, isoproterenol infusion was continuously infused for three days. The patient was free of VF during this period and the ECG remained normal. Weaning from mechanical ventilation occurred on day 2. Echocardiogram and cardiac MRI examination excluded any structural heart disease and left ventricular ejection fraction was normalized. Catheterization revealed no significant coronary artery disease. Normal troponine, CPK enzymes and normal cardiac MRI excluded myocarditis. After written informed consent was obtained, an electrophysiological study was done off isoproterenol. No ventricular or supraventricular tachycardia could be induced by programed right ventricular and atrial stimulation. Atrioventricular conduction was normal, as were tests of sinus node function. The right ventricle refractory period was $210 \mathrm{~ms}$. The flecainide provocation test revealed no Brugada syndrome nor any repolarization abnormalities.

An implantable single chamber cardioverter defibrillator was implanted. Interestingly, upon discharge from the hospital the patient had no ventricular arrhythmias, while 
Fig. 2 (a) and (b) Onset of two ventricular arrhythmias. The sequence is always the same: a premature beat with major early repolarization $(*)$ and long coupling interval $(450 \mathrm{~ms})$ was followed by a premature ventricular beat $(\boldsymbol{\nabla})$ left bundle branch block pattern and left axis with short coupling interval (320 ms) initiating VF. Episodes of VF was closely linked to the $\mathrm{J}$ point elevation
(A)

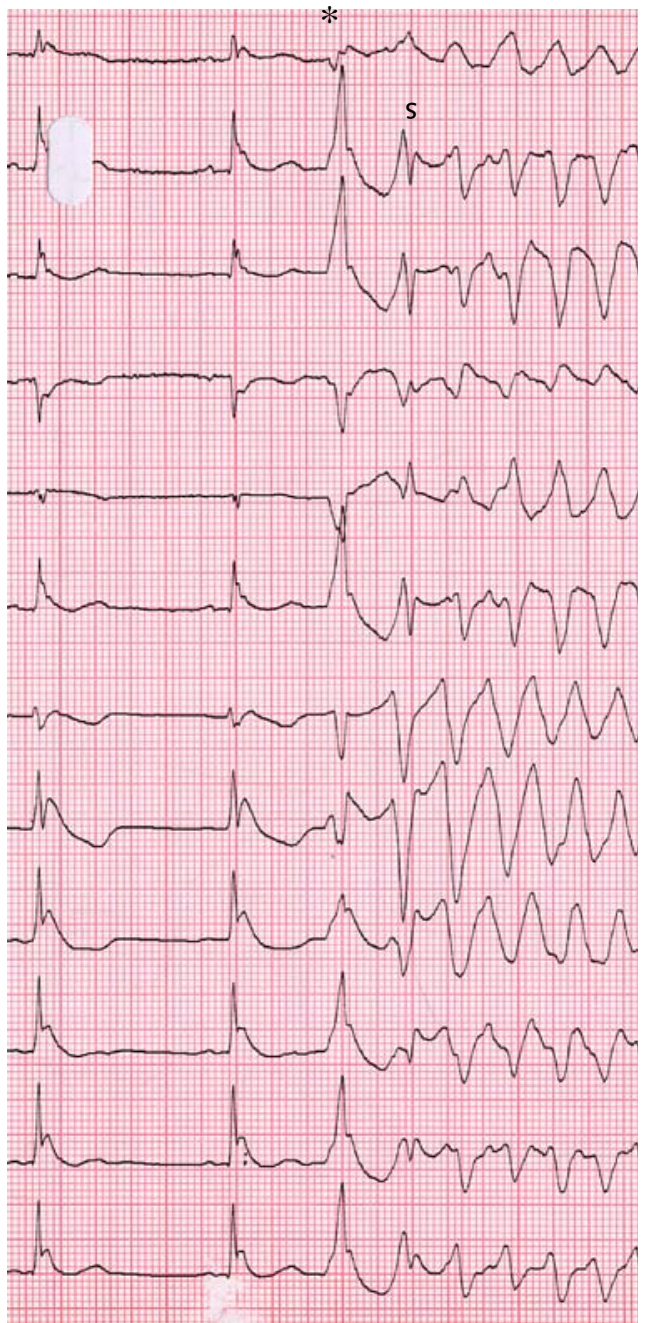

$25 \mathrm{~mm} / \mathrm{sec}$
(B)

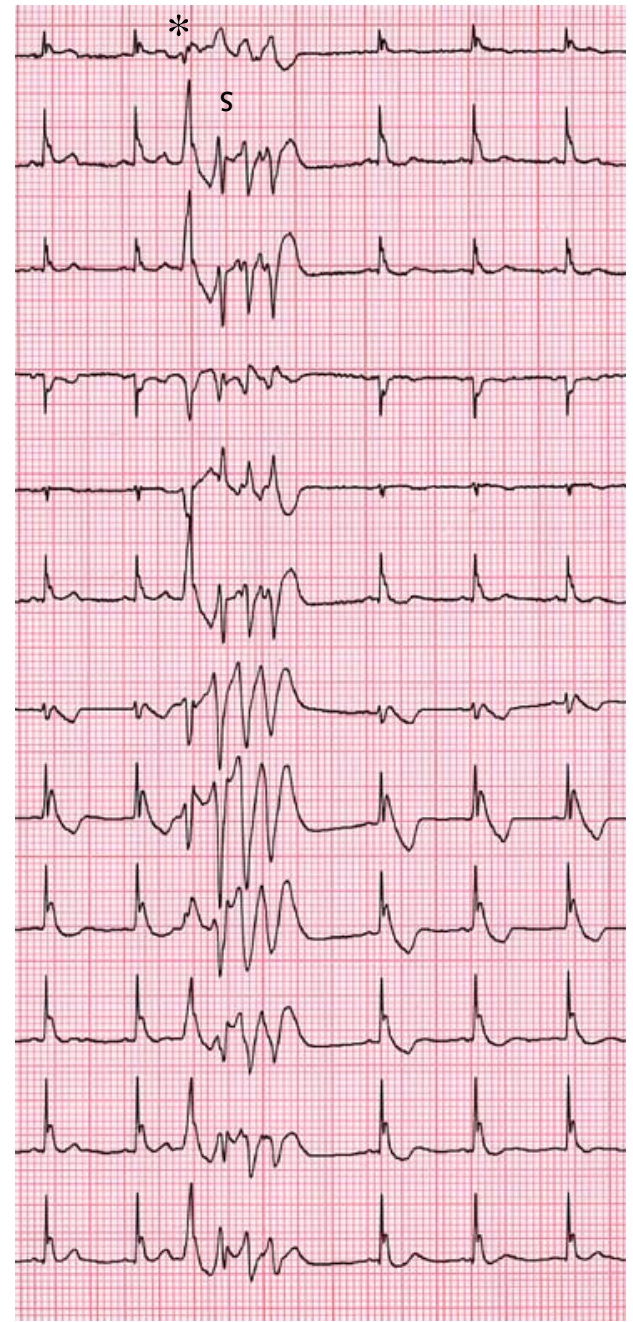

$12.5 \mathrm{~mm} / \mathrm{sec}$ the $\mathrm{J}$ point and ST segment were normalized without antiarrhythmic drugs. The back up pacing was programmed at 70 beats/min (VVI). Over a follow-up of six months, the patient did not present any new syncope and no appropriate therapy was delivered by the device. In the ICD memory, the patient was not chronically paced ( $7 \%$ of paced beats).

We would like to highlight that early repolarization, a common electrocardiographic finding usually considered benign, was in this case responsible for ventricular electrical storm. From the electrocardiographic point of view, our case is remarkable for the evolution of the $\mathrm{J}$ wave elevation between the first ECG (Fig. 1(a)) and the ECG performed just before VF, which resembled the Osborn wave (Fig. 1(c)). Early repolarization affects about $1-5 \%$ of the general population and is defined by the elevation of the $\mathrm{J}$ wave at least $1 \mathrm{~mm}$ above the baseline [1]. Haissaguerre et al recently showed that incidence of early repolarization was higher in patients suffering from unexplained ventricular fibrillation than in a control population (31\% versus $5 \%$ [3]. Early repolarization was mainly present in the inferior and lateral leads. Moreover, in the population exhibiting primary ventricular fibrillation and implanted with a cardiac defibrillator the recurrence of ventricular fibrillation was more frequent in patients with early repolarization than in those without [3]. This suggested potential arrhythmogenicity of early repolarization, which could be the underlying mechanism in a subgroup of idiopathic VF. Previous experimental studies have evoked the potential arrhythmogenicity of the early repolarization [2]. In rare idiopathic VF a prominent $J$ wave (Osborn wave) in the inferior leads has also been reported [6]. In our case the repolarization abnormalities and the ventricular arrhythmias were dependent on the sinus rate: Low heart rate was linked to major early repolarization and 
Fig. 3 Electrocardiographic patterns before and during isoproterenol infusion. Before isoproterenol $\mathrm{J}$ point and ST elevations were observed in leads V2-V6. During isoproterenol J point and ST segment were isoelectrical while heart rate was $110 / \mathrm{min}$

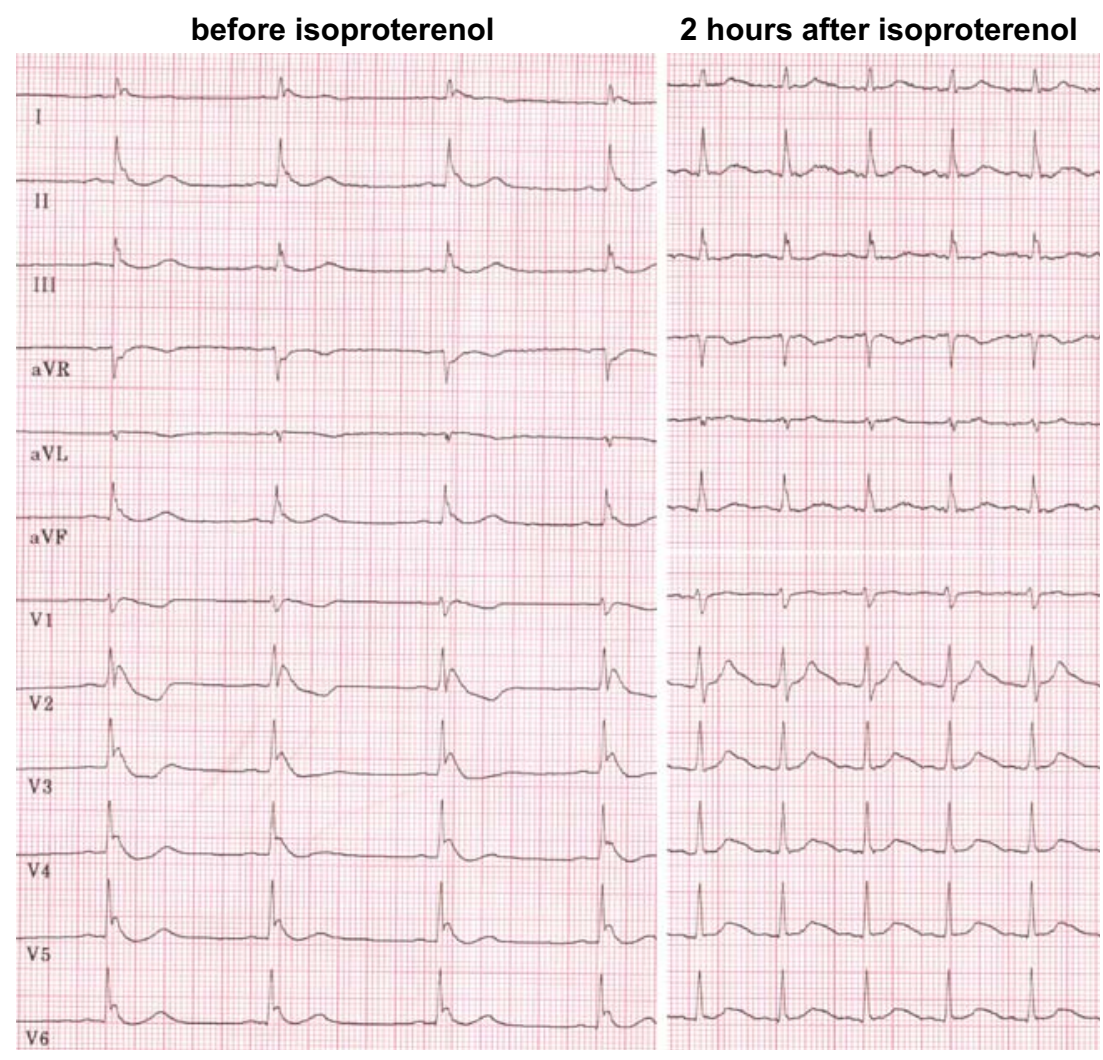

ventricular fibrillation, whereas normalization of the ECG and the disappearance of VF were obtained by accelerating the sinus rhythm. The causal factor of the incessant arrhythmias could be the increase in vagal tone during gastro-enteritis [7] and hypokalemia. The exacerbation of the vagal tone or slowing of the heart rate tended to increase the transmural voltage gradient responsible for the development of a vulnerable window during which a premature beat can induce a re-entrant arrhythmia [1]. The increase in transmural voltage gradient could also be the mechanism of the premature beat itself (phase 2 reentry) as proposed by Antzelevitch in Brugada syndrome [8]. In fact, the transmural voltage gradient is in part generated by the heterogeneity of the distribution of the transient outward potassium current (Ito) between epicardium and endocardium. Inactivation and reactivation rates of Ito are sensitive to the heart rate. We hypothesized that isoproterenol could play a decisive role by accelerating heart rate and directly affecting the myocardium (enhancing the inward calcium current) to eliminate the transmural voltage gradient as in Brugada syndrome $[9,10]$. Another possible method to accelerate heart rate should be temporary rapid cardiac pacing. This would have helped in analyzing the possible mechanisms of isoproterenol (cardiac acceleration) versus direct effect of isoproterenol on membrane currents. Hypokalemia might also favor the occurrence of VF, but analysis of the ECG repolarization showed normal QT interval, while infusion of potassium and magnesium was rapidly started with no effect on the occurrence of VF. Moreover, Ito current is not modified by extracellular $\mathrm{K}+$ concentration [11]. To prevent recurrence of electrical storm, it may be tested a chronic treatment by oral quinidine that previously proved its efficiency in Brugada syndrome and idiopathic ventricular fibrillation $[10,12]$. The efficiency of quinidine in these primary electrical cardiac diseases is attributed to the normalization of the transmural voltage gradients [10].

In the presence of electrical storm, radiofrequency ablation of ectopic beats triggering ventricular fibrillation is another therapeutic possibility [3]. This assumes limited number of different premature beats. In our case VF was always initiated by the same premature ventricular beat and radiofrequency ablation could be proposed if the patient comes back with a new electrical storm. Finally, in clinical practice isoproterenol infusion is simple and efficient therapy to eliminate recurrent VF in patients with early repolarization.

Open Access This article is distributed under the terms of the Creative Commons Attribution Noncommercial License which permits any noncommercial use, distribution, and reproduction in any medium, provided the original author(s) and source are credited. 


\section{References}

1. Gussak, I., \& Antzelevitch, C. (2000). Early repolarization syndrome: clinical characteristics and possible cellular and ionic mechanisms. Journal of Electrocardiology, 33, 299-309.

2. Boineau, J. P. (2007). The early repolarization variant-normal or a marker of heart disease in certain subjects. Journal of Electrocardiology, 40, 3.e11-3.e16.

3. Haïssaguerre, M., Derval, N., Sacher, F., et al. (2008). Sudden death associated with early repolarization. The New England Journal of Medicine, 358, 2016-2023.

4. Nam, G. B., Kim, Y. H., \& Antzelevitch, C. (2008). Augmentation of $\mathrm{J}$ waves and electrical storm in patients with early repolarization. The New England Journal of Medicine, 358, 2078-2079.

5. Rosso, R., Kogan, E., \& Belhassen, B. (2008). J-point elevation in survivors of primary ventricular fibrillation and matched control subjects. Journal of the American College of Cardiology, 52, 1231-1238.

6. Kalla, H., Yan, G. X., \& Marinchak, R. (2000). Ventricular fibrillation in a patient with prominent J (Osborn) waves and ST segment elevation in the inferior electrocardiographic leads: a Brugada syndrome variant? Journal of Cardiovascular Electrophysiology, 11, 95-98.
7. Maury, P., Couderc, P., Delay, M., Boveda, S., \& Brugada, J. (2004). Electrical storm in Brugada syndrome successfully treated using isoproterenol. Europace, 6, 130-133.

8. Antzelevitch, C. (2001). The Brugada syndrome: ionic basis and arrhythmias mechanisms. Journal of Cardiovascular Electrophysiology, 12, 268-270.

9. Watanabe, A., Kusano, K. F., \& Morita, H. (2006). Low dose isoproterenol for repetitive ventricular arrhythmia in patients with Brugada syndrome. European Heart Journal, 27, 15791583.

10. Ohgo, T., Okamura, H., \& Noda, T. (2007). Acute and chronic management in patients with Brugada syndrome associated with electrical storm of ventricular fibrillation. Heart Rhythm, 4, 695700 .

11. Hirota, M., Ohtani, H., \& Hanada, E. (2000). Influence of extracellular $\mathrm{K}^{+}$concentrations on quinidine-induced $\mathrm{K}^{+}$current inhibition in rat ventricular myocytes. The Journal of Pharmacy and Pharmacology, 52, 99-105.

12. Belhassen, B., Viskin, S., Fish, R., Glick, A., Setbon, I., \& Eldar, M. (1999). Effects of electrophysiologic-guided therapy with class IA antiarrhythmic drugs on the long-term outcome of patients with idiopathic ventricular fibrillation with or without the Brugada syndrome. Journal of Cardiovascular Electrophysiology, 10, 1301-1312. 